\title{
Consecuencias jurídicas de los accidentes durante las clases de tenis
}

\author{
Alejandro Valiño \\ Universidad de Valencia, Valencia, España
}

\section{RESUMEN}

La concentración de personas en una pista de tenis en las clases colectivas es un factor de riesgo de producción de daños personales. El objeto de este artículo es delimitar a quién es imputable y en qué circunstancias. Siendo que nuestros lectores son de distintas nacionalidades, sin perjuicio de puntuales referencias a ordenamientos jurídicos concretos, significaremos los principios esenciales sobre la materia que, en cuanto trasladables a cualquier ámbito territorial, se contienen en el Proyecto de un Marco Común de Referencia para el Derecho privado europeo (DCFR) ${ }^{1}$ o en los Principios de Derecho europeo de la responsabilidad civil $^{2}$.
Palabras clave: accidentes, responsabilidad, sesiones, daños

Recibido: 04 Mayo 2018

Aceptado: 15 Junio 2018

Autor correspondiente:

Alejandro Valiño

Universidad de Valencia,

Valencia, España.

Correo electrónico:

alejandro.valino@uv.es

\section{ORGANIZACIÓN, DIRECCIÓN Y EJECUCIÓN DE} PROGRAMAS DE ENSEÑANZA DE TENIS

Estas tres funciones, junto con la evaluación de los resultados, constituyen la esencia de un programa de enseñanza de tenis ${ }^{3}$. Pueden concurrir en una o en varias personas. En el primer caso, estaremos ante el entrenador autónomo que 'monta' su escuela de tenis en una instalación, organizando los grupos, impartiendo las clases, percibiendo de sus alumnos una retribución y, en su caso, pagando por el uso de las pistas a su titular. En el segundo caso, una entidad deportiva directamente o una empresa externa de servicios deportivos organiza la escuela de tenis, designando a un director y contratando técnicos encargados de impartir las clases.

¿A quién puede exigírsele responsabilidad si por un accidente se producen daños? Para responder a esta cuestión hay que partir de la relación jurídica que surge detrás de las clases de tenis.

${ }^{1}$ Draft Common Frame of Reference, Principles, Definitions and Model Rules of European Private Law, accesible en https://www.degruyter.com/view/product/41776. ${ }^{2}$ Principles of European Tort Law, European Group on Tort Law, accesible en http://civil.udg.edu/php/biblioteca/items/298/PETLSpanish.pf.

${ }^{3}$ Sobre el particular, vid. VALIÑO ARCOS, A. (2017). Gerentes y directores de organizaciones deportivas dedicadas al tenis. En: E-Coach. Revista Electrónica del Técnico de Tenis 9, 48-56.

\section{NATURALEZA JURÍDICA DE LAS CLASES DE TENIS}

La enseñanza del tenis, como actividad retribuida, encaja en el contrato de servicios. El DCFR lo define como un contrato en el que el proveedor del servicio (the service provider) se compromete a prestar un servicio (undertakes to supply a service) al cliente (the client), a cambio de un precio (in exchange for a price $)^{4}$. El proveedor del servicio sería el club, la empresa externa o el entrenador, mientras que el cliente sería el alumno o sus familiares. La obligación del proveedor no es de resultado, pues, como actividad aleatoria que es, nadie suele comprometerse a un preciso objetivo deportivo concreto, como ganar Wimbledon o ser número 1 del ranking ATP. Su obligación es de medios, esto es, ofrecer un servicio de calidad conforme con las técnicas y métodos de enseñanza adecuados para procurar la satisfacción, aprendizaje o mejora del nivel de los alumnos, atendida su edad, aptitud técnica e inquietudes.

${ }^{4}$ Art. IV.C.-1:101 del DCFR. 


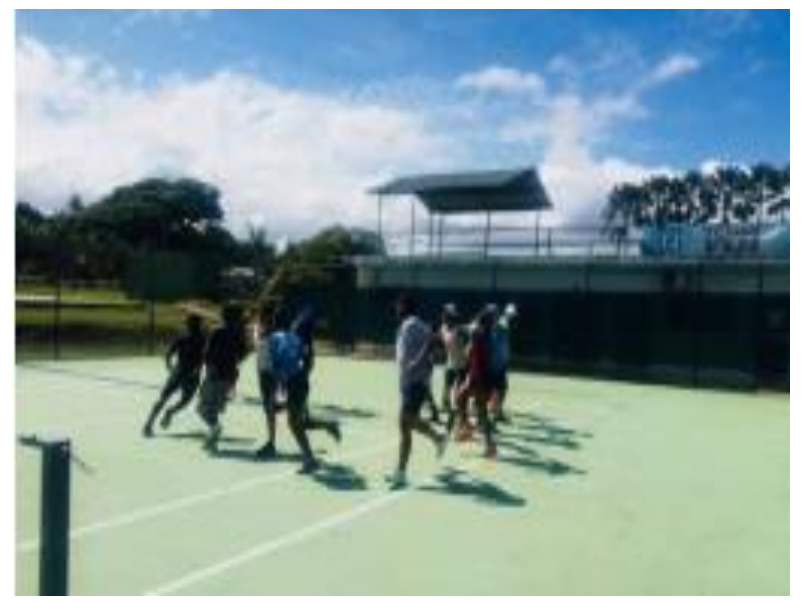

\section{DISTINCIÓN ENTRE RESPONSABILIDAD CONTRACTUAL} Y EXTRACONTRACTUAL

El entrenador debe proceder con la máxima diligencia a fin de no defraudar las expectativas del alumno y minimizar el riesgo de que se produzcan accidentes durante sus clases. Queda así vislumbrada la distinción entre responsabilidad contractual y extracontractual.

La primera será exigible por incumplimiento o cumplimiento deficiente o tardío de la prestación del entrenador: ausencia, impuntualidad, desidia o manifiesta incompetencia en la conducción de las clases son comportamientos que encajarían en este ámbito 5 .

La segunda entra en juego cuando se producen daños durante las clases por causa externa a la prestación del servicio, por ejemplo, por un mal estado de la cancha.

Estas dos modalidades de responsabilidad son exigibles mediante medios judiciales distintos, si bien, no siendo siempre nítidas las fronteras entre un tipo y otro, no es extraño su ejercicio acumulado, teniendo en la mayor parte de los ordenamientos jurídicos plazos de prescripción diferentes ${ }^{6}$.

\section{DAÑOS INDEMNIZABLES Y DAÑOS NO INDEMNIZABLES}

Conviene, sin embargo, precisar que no todo daño desencadena responsabilidad. El deporte lleva inherentes

${ }^{5}$ Art. IV.C.-2:105(1) del DCFR: "el proveedor del servicio debe realizar el servicio con el cuidado y la habilidad que un proveedor razonable de servicios podría ejercer bajo las circunstancias". Para un estándar del entrenador ideal, vid. CRESPO CELDA, M. (1994). Cómo ser un buen entrenador de tenis. En: Workshop Regional de la ITF para Entrenadores. Montevideo, accesible en http://www.miguelcrespo.net/articulos/Crespo.\%20Como\%20ser\%20un\%20buen\%2 Oentrenador\%20de\%20tenis.\%20Uruguay,\%201994.pdf.

${ }^{6}$ En el Código Civil español, la acción para exigir responsabilidad contractual prescribe a los cinco años, mientras que la acción para exigir responsabilidad extracontractual prescribe en el plazo de un año. ciertos riesgos, cuyas consecuencias dañosas han de ser asumidos por el deportista, por ejemplo, lesiones leves producto de una caída o del contacto accidental con un compañero de clase. Todo depende de quién sea el accidentado y qué grado de conocimiento tenga sobre el estado de la cancha o sobre las condiciones de la práctica del deporte.

\section{Deficiente estado de conservación de la pista}

Los tribunales en España han desestimado reclamaciones por caídas accidentales que sólo muy remotamente podrían deberse al estado de la pista, por ejemplo, por tropiezos en las canchas de tierra batida ante la ligerísima elevación de las líneas respecto a la superficie, que han provocado lesiones leves ${ }^{7}$. En cambio, si la causa de un daño extraordinario, como una lesión grave o, incluso, la muerte es la patente falta de mantenimiento de la pista, podría exigirse responsabilidad extracontractual del organizador de las clases y/o del titular de la instalación, según cuál sea la causa eficiente de su producción. Por ejemplo, si el mal estado de la moqueta propicia la caída de un alumno con tan mala suerte que su cabeza impacta en el suelo, provocándole la muerte, la posibilidad de obtener una indemnización está servida en la medida en que se ha producido un daño patrimonialmente indemnizable imputable a una falta de conservación de la pista, pudiéndose establecer el nexo causal entre esa falta de mantenimiento y el daño extraordinario causado, que es imputable al titular de la instalación en la que tienen lugar las clases $^{8}$.

Impracticabilidad de la pista por las condiciones meteorológicas

Cuando el riesgo dependa de la puntual impracticabilidad de la pista tras unas copiosas lluvias, quién sea el accidentado puede ser relevante para la estimación de la reclamación. Así, a los entrenadores y deportistas expertos puede presuponérseles un conocimiento sobre el estado de la pista que, sin embargo, no concurre en alumnos principiantes. De ahí que las lesiones por caídas accidentales den lugar en el primer supuesto a la aplicación de la teoría de la asunción del riesgo por parte del lesionado ${ }^{9}$, mientras que en el segundo está servida la posibilidad de reclamar contra el organizador de las clases o el titular de la instalación que ha puesto innecesariamente en riesgo a sus alumnos.

\section{El riesgo por condiciones climatológicas extremas}

Durante el verano y en zonas donde es habitual que las tempe-

${ }^{7}$ Para un caso semejante, vid. Sentencia de la Audiencia Provincial de Málaga (Sección 4a) $n^{\circ} 896 / 2004$, de 9 de diciembre

${ }^{8}$ Un caso real acaecido en España en una pista de pádel fue resuelto por la Sentencia de la Audiencia Provincial de Valencia (Sección 6a) no 469/2012, de 23 de julio, condenando al club deportivo por la muerte accidental de un deportista por ser el estado de conservación de la moqueta de la cancha el que provocó la caída de tan fatal desenlace.

'La Sentencia de la Audiencia Provincial de Cádiz de 4 de enero de 1999, citada por CASADO ANDRÉS, B. (2017). El tenis: ¿un deporte de riesgo? En: Diario La Ley $n^{\circ} 9023$, Sección Tribuna, 5(13), a propósito de la lesión de un entrenador de tenis. 
raturas superen los 30 grados, el riesgo de que se produzcan accidentes se incrementa notablemente. Por ello, un entrenador prudente debe programar sus clases en aquellas horas del día más propicias para evitar daños personales. Esta misma idea es predicable en el ámbito de las competiciones, por lo que sus organizadores deben abstenerse de programar partidos en las horas de máximo calor y humedad ${ }^{10}$.

\section{El riesgo por imprudencia del entrenador en la ejecución de la prestación}

Un material inadecuado o un excesivo número de alumnos es fuente potencial de daños imputables al entrenador. El uso de pelotas no acordes con el nivel de los alumnos dificulta su control e incrementa el nivel de riesgo. Lo mismo sucede con los encontronazos o impactos que se dan accidentalmente entre los alumnos, por ejemplo durante los tiempos en que guardan la cola o en que están dedicados a la recogida de pelotas. Estos accidentes pueden desencadenar la responsabilidad del organizador de las clases si no adopta las oportunas medidas de prevención para su evitación. Así sucedió en un club de tenis en Murcia (España), en el que un niño tuvo que someterse a varias intervenciones quirúrgicas en uno de sus ojos tras ser golpeado por la raqueta de un compañero. La sentencia consideró responsable al Club de Tenis donde, a cargo de un monitor titulado contratado por la entidad, se impartía la clase en la que se produjo el desgraciado accidente mientras los niños recogían pelotas, sirviéndose de las raquetas a modo de bandeja. El criterio de imputación fue la culpa o negligencia por falta de vigilancia y control sobre los niños ${ }^{11}$.

\section{PRESUPUESTOS DE IMPUTACIÓN DEL DAÑO}

Asumir responsabilidad por los daños causados durante las clases depende de la diligencia de quien las organiza. Mantener la cancha en buen estado, emplear pelotas de colores cuando los alumnos no tienen demasiado control y no sobrecargar la clase de alumnos son medidas que contribuyen a prevenir $\mathrm{O}$, al menos, a reducir considerablemente los riesgos. Y lo mismo puede decirse de la elección y vigilancia de los entrenadores contratados, asegurándose el organizador de que la metodología y ejercicios que se aplican no ponen en peligro la seguridad y salud de los alumnos ${ }^{12}$. Si todas estas cautelas se observan $y$, pese a ello, los daños se producen, a menos que sean extraordinarios, han de ser asumidos por el deportista, pues, si es indudable que el deporte beneficia por

${ }^{10} \mathrm{El}$ art. 8.5.h) del Reglamento de Justicia Deportiva de la Federación de Tenis de la Comunidad Valenciana tipifica como infracción muy grave de los órganos arbitrales "programar en época estival partidos entre las 14 y las $17 \mathrm{~h}$. cuando la temperatura prevista supere, según la Agencia Nacional de Meteorología, los 35 grados y los participantes en el torneo o competición sean menores de edad, sin que en modo alguno puedan programarse partidos más allá de las 21 horas ni autorizarse la disputa de partidos programados con anterioridad, pero que por retraso no hayan dado comienzo antes de las 22 horas, los cuales habrán de aplazarse para no antes de las $10 \mathrm{~h}$. del día siguiente".

${ }^{11}$ Sentencia de la Audiencia Provincial de Murcia (Sección 4a) $n^{\circ}$ 458/2013, de 18 de julio.

${ }^{12}$ El artículo 6:102 (responsabilidad por los auxiliares) de los Principios de Derecho europeo de la responsabilidad civil dispone que "una persona responde por el daño causado por sus auxiliares en el ejercicio de sus funciones siempre que éstos hayan violado el estándar de conducta exigible".

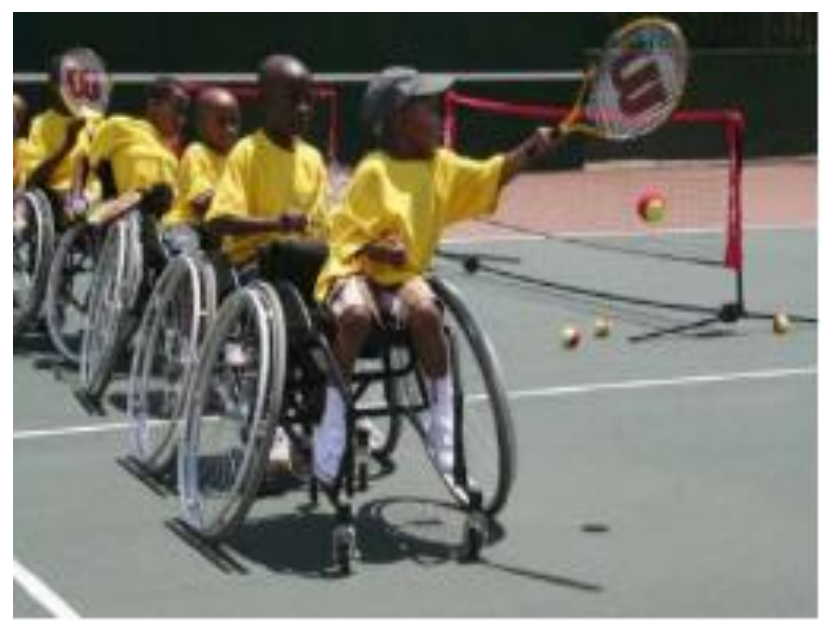

principio la salud física y mental de quien lo practica, no está exento de ciertos riesgos ${ }^{13}$.

En los deportes de raqueta, por la propia disposición alejada de los jugadores, no son frecuentes los impactos. Pero el riesgo se acentúa en los partidos de dobles y, sobre todo, en las clases colectivas de tenis por la no desdeñable concentración de alumnos en un reducido espacio. En el primer supuesto, cobra plena aplicación la teoría de la asunción del riesgo. En el segundo, la naturaleza retribuida del servicio en que las clases consisten hace presumir de entrada la culpabilidad del organizador, que incluso a veces puede haber contribuido a la intensificación del riesgo, por ejemplo, acogiendo mayor número de alumnos de lo que sería prudente y razonable o empleando pelotas altamente desgastadas que incrementa considerablemente los niveles de riesgo ${ }^{14}$.

\section{LA IMPORTANCIA DE CONTAR CON UN SEGURO DE RESPONSABILIDAD CIVIL}

Aunque es ya una exigencia normativa en muchos ordenamientos jurídicos ${ }^{15}$, los entrenadores de tenis, altamente expuestos a los riesgos inherentes a las clases colectivas, han de suscribir una póliza de seguro de responsabilidad civil. Así, a cambio del pago de una prima, será la compañía de seguros quien tenga que hacer frente a una indemnización si algún alumno sufre daños por un accidente durante las clases.
${ }^{13}$ La teoría de la asunción del riesgo se formuló por primera vez en España en la Sentencia del Tribunal Supremo, Sala de lo Civil, de 22 de octubre de 1992 a propósito de un accidente habido en un partida de pelota en la que uno de los jugadores perdió un ojo tras un pelotazo accidental de un compañero, quien no fue considerado responsable al tratarse de un lance del juego en el que no intervino por su parte dolo ni culpa, ya que no se apartó de los usos propios de la práctica de este deporte.

${ }^{14}$ Sobre la cuestión de los riesgos en el deporte, vid. VERDERA SERVER, R. (2003). Una aproximación a los riesgos del deporte. En: InDret. Revista para el análisis del Derecho, 1, 1-19, accesible en http://www.indret.com/ pdf/116_es.pdf y CASADO ANDRÉS, B. (2017). El tenis: ¿un deporte de riesgo). En: Diario La Ley, n 9023, Sección Tribuna, 3 (13).

${ }^{15}$ En España es una constante en prácticamente todas las Leyes autonómicas del deporte, estando tipificada su contravención como una infracción administrativa sancionable con fuertes multas económicas. 


\section{CONCLUSIÓN}

La enseñanza colectiva del tenis, al concentrarse un mayor número de jugadores en un reducido espacio, supone unos riesgos adicionales que obligan al entrenador de tenis a ser extremadamente diligente en cuanto al número de alumnos que tienen cabida en una misma clase, al material escogido cuando la destreza de los alumnos sea escasa y a las horas del día en que programa sus clases si las condiciones climatológicas son extremas. Aunque no es frecuente que se produzcan daños en las clases de tenis, la prudencia aconseja a los clubes y entrenadores suscribir una póliza de seguro de responsabilidad civil que reduzca al máximo la posibilidad de sufrir un notable quebranto económico si son objeto de reclamaciones judiciales por la producción de daños durante las clases de tenis que organizan.

\section{REFERENCIAS}

Casado Andrés, B. (2017). El tenis: ¿un deporte de riesgo? En: Diario La Ley, nº 9023, Sección Tribuna, 1-13.

Crespo Celda, M. (1994). Cómo ser un buen entrenador de tenis. En: Workshop Regional de la ITF para Entrenadores. Montevideo, accesible http://www.miguelcrespo.net/articulos/Crespo.\%20Como\%20se r\%20un\%20buen\%20entrenador\%20de\%20tenis.\%20Uruguay, \%201994.pdf.

Valiño Arcos, A. (2017). Gerentes y directores de organizaciones deportivas dedicadas al tenis. En: E-Coach - Electronic magazine of the Tennis Coach, 29 (9), 40-57.
Verdera Server, R. (2003). Una aproximación a los riesgos del deporte. En: InDret. Revista para el análisis del Derecho, 1, 1-19, accesible en http://www.indret.com/pdf/116_es.pdf.

CONTENIDO ITF ACADEMY RECOMENDADO (HAZ CLICK ABAJO)

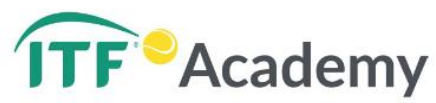

Derechos de Autor (c) 2018 Alejandro Valiño

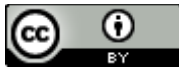

Este texto está protegido por una licencia CreativeCommons $\underline{4.0}$.

Usted es libre para Compartir — copiar y redistribuir el material en cualquier medio o formato- y Adaptar el documento — remezclar, transformar y crear a partir del material- para cualquier propósito, , incluso para fines comerciales, siempre que cumpla la condición de:

Atribución: Usted debe dar crédito a la obra original de manera adecuada, proporcionar un enlace a la licencia, e indicar si se han realizado cambios. Puede hacerlo en cualquier forma razonable, pero no de forma tal que sugiera que tiene el apoyo del licenciante o lo recibe por el uso que hace de la obra.

Resumendelicencia - Textocompletodelalicencia 